\title{
IMAGE ANALYSIS FOR COSMOLOGY: RESULTS FROM THE GREAT10 STAR CHALLENGE
}

\author{
T. D. Kitching ${ }^{1}$, B. Rowe ${ }^{2,3}$, M. Gill ${ }^{4,5,6}$, C. Heymans ${ }^{1}$, R. Massey ${ }^{7}$, D. Witherick ${ }^{2}$, F. Courbin ${ }^{8}$, K. GeOrgatzis 9,10 ,

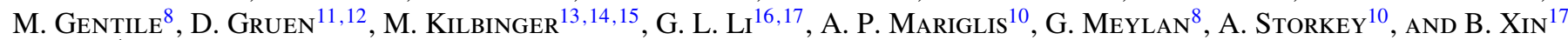 \\ ${ }^{1}$ Mullard Space Science Laboratory, University College London, Holmbury St. Mary, Dorking, Surrey RH5 6NT, UK; t.kitching@ucl.ac.uk \\ ${ }^{2}$ Department of Physics and Astronomy, University College London, Gower Street, London WC1E 6BT, UK \\ ${ }^{3}$ California Institute of Technology, 1200 East California Boulevard, Pasadena, CA 91125, USA \\ ${ }^{4}$ Center for Cosmology and AstroParticle Physics, Physics Department, The Ohio State University, Columbus, OH, USA \\ ${ }^{5}$ Kavli Institute for Particle Astrophysics \& Cosmology, Stanford, CA, USA \\ ${ }^{6}$ Centro Brasileiro de Pesquisas Físicas, Rio de Janeiro, RJ, Brazil \\ ${ }^{7}$ Institute for Computational Cosmology, Durham University, South Road, Durham DH1 3LE, UK \\ ${ }^{8}$ Laboratoire d'Astrophysique, École Polytechnique Federale de Lausanne (EPFL), Switzerland \\ ${ }^{9}$ Department of Information and Computer Science, Aalto University, P.O. Box 15400, FI-00076 Aalto, Finland \\ ${ }^{10}$ School of Informatics, University of Edinburgh, 10 Crichton Street, Edinburgh EH8 9AB, UK \\ ${ }^{11}$ Department of Physics and Astronomy, 209 South 33rd Street, University of Pennsylvania, Philadelphia, PA 19104, USA \\ ${ }^{12}$ University Observatory Munich, Scheinerstrasse 1, D-81679 Munich, Germany \\ ${ }^{13}$ Excellence Cluster Universe, Boltzmannstr. 2, D-85748 Garching, Germany \\ ${ }^{14}$ Universitäts-Sternwarte, Ludwig-Maximillians-Universität München, Scheinerstr. 1, D-81679 München, Germany \\ ${ }^{15}$ CEA Saclay, Service d'Astrophysique (SAp), Orme des Merisiers, Bât 709, F-91191 Gif-sur-Yvette, France \\ ${ }^{16}$ Purple Mountain Observatory, 2 West Beijing Road, Nanjing 210008, China \\ ${ }^{17}$ Department of Physics, Purdue University, 525 Northwestern Avenue, West Lafayette, IN 47907, USA \\ Received 2012 October 6; accepted 2013 February 6; published 2013 March 5
}

\begin{abstract}
We present the results from the first public blind point-spread function (PSF) reconstruction challenge, the GRavitational lEnsing Accuracy Testing 2010 (GREAT10) Star Challenge. Reconstruction of a spatially varying PSF, sparsely sampled by stars, at non-star positions is a critical part in the image analysis for weak lensing where inaccuracies in the modeled ellipticity $e$ and size $R^{2}$ can impact the ability to measure the shapes of galaxies. This is of importance because weak lensing is a particularly sensitive probe of dark energy and can be used to map the mass distribution of large scale structure. Participants in the challenge were presented with 27,500 stars over 1300 images subdivided into 26 sets, where in each set a category change was made in the type or spatial variation of the PSF. Thirty submissions were made by nine teams. The best methods reconstructed the PSF with an accuracy of $\sigma(e) \approx 2.5 \times 10^{-4}$ and $\sigma\left(R^{2}\right) / R^{2} \approx 7.4 \times 10^{-4}$. For a fixed pixel scale, narrower PSFs were found to be more difficult to model than larger PSFs, and the PSF reconstruction was severely degraded with the inclusion of an atmospheric turbulence model (although this result is likely to be a strong function of the amplitude of the turbulence power spectrum).
\end{abstract}

Key words: atmospheric effects - cosmology: observations - methods: data analysis - techniques: image processing

Online-only material: color figures

\section{INTRODUCTION}

In this paper we present the results from the GRavitational 1Ensing Accuracy Testing 2010 (GREAT10) Star Challenge. GREAT10 was an image analysis challenge for cosmology that focused on the task of measuring the shapes of distant galaxies. Light from distant galaxies is deflected during its journey to us via gravitational lensing, and the images appear distorted into characteristic patterns (Hu 1999; Bartelmann \& Schneider 2001). The amount of distortion depends on the intervening distribution of matter (including dark matter) and the geometry of spacetime (which is currently governed by dark energy). Such measurements thus probe directly the invisible dark sector and the fundamental nature of gravity - see reviews by Albrecht et al. (2006), Réfrégier (2003), Hoekstra \& Jain (2008), Massey et al. (2010), and Weinberg et al. (2012).

All real imaging data are necessarily seen after convolution with (i.e., blurring by) a telescope's point-spread function (PSF). The PSF arises from the finite aperture of the telescope, charge diffusion within digital detectors, any imperfect elements along the optical path, and turbulence in the Earth's atmosphere (unless the telescope is in space). This increases the size of faint galaxies, and can spuriously change their ellipticity by an order of magnitude more than gravitational lensing (Bernstein
\& Jarvis 2002; Hoekstra 2004; Paulin-Henriksson et al. 2008, 2009; Massey et al. 2013). To recover the shape of the galaxy after only cosmological effects, it is necessary to (1) model the PSF and (2) somehow correct for its effect on the images of galaxies. The second half of this task has been widely addressed by teams analyzing individual surveys and as a vital community effort through the public Shear TEsting Programme (STEP) (Heymans et al. 2006; Massey et al. 2007), the GRavitational lEnsing Accuracy Testing (GREAT) galaxy challenges (Bridle et al. 2010; Kitching et al. 2012b), and the Mapping Dark Matter challenge (Kitching et al. 2012c). The first task (modeling the PSF) has so far only been investigated internally within teams (e.g., Bacon et al. 2003; Hoekstra et al. 2004; van Waerbeke et al. 2005; Rhodes et al. 2007; Schrabback et al. 2010; Hoekstra 2004; Rowe 2010; Jarvis \& Jain 2005; Bergé et al. 2012). Here we present the results of the first blind, public trial of methods to model and interpolate the PSF of a typical astronomical telescope.

The PSF in an astronomical image can be measured from stars that happen to fall inside the field of view. Stars are so small that they are intrinsically point-like, and adopt the size and shape of the telescope's PSF. However, the PSF typically varies across the field of view, and stars only sparsely cover the extragalactic sky (Jarvis \& Jain 2005; Jain et al. 2006; Heymans 
et al. 2012; Chang et al. 2012). It is therefore necessary to model the shapes of stars, then interpolate their shapes to the locations of galaxies (where there is necessarily not a bright star because otherwise the galaxy could not be seen). In practice the PSF also varies as a function of the wavelength of observed light, due to diffraction, reflection, and transmission effects in the telescope optics, filters, and CCDs and so must also be interpolated from the colors of the stars to the colors of the galaxy (Cypriano et al. 2010; Voigt et al. 2012; Plazas \& Bernstein 2012). Color dependence is an important second order effect but in this paper we do not address this, focusing only on the primary changes in PSFs.

We simulated the spatial variation in the PSF of generic but realistic ground-, balloon-, and space-based telescopes (Kitching et al. 2012a, and see www.greatchallenges.info). We realized a large suite of sparse stellar fields in these different observing regimes, and publicly released most of the star images. Entrants were asked to then reconstruct the images of the missing stars on a pixel grid, at pre-defined locations. The performance of each entry was measured in real time using a single number, the "quality factor," which was designed to provide a crude ranking such that it could not be reverse-engineered to reveal the full solutions. In this paper, we analyze in detail the quantitative performance of 12 distinct algorithms submitted to model and interpolate the simulated PSFs. In particular we quantify how well the ellipticity and size of a spatially varying PSF can be reconstructed in a blind challenge.

This paper is organized as follows. In Section 2, we describe the simulations and competition in detail. In Section 3, we present results. We discuss and conclude in Section 4.

\section{METHOD}

In this section we describe the simulations and the competition. For a full exposition of the background of the Star Challenge, see Kitching et al. (2012a).

\subsection{Simulation Structure}

In the simulations we aimed to generate simplified representations of possible observing scenarios and telescopes, such that through analysis we could make general statements about how methods perform in a coarse-grained sense in each of these categories.

The simulations contained two possible types of PSF function: a Moffat function (Moffat 1969) and an Airy disk, parameterized by a FWHM size. To simulate diffraction spikes caused by obscuration of the telescope pupil the intensity distributions of these functions were optionally combined with single-slit diffraction intensity patterns, approximating the effects of rectangular obscurations in the pupil plane as would be caused by struts supporting a secondary mirror. The dimensions of these single-slit obscurations were chosen to produce simulated PSFs of reasonable realism on visual inspection; for the Airy disk this corresponded to a strut obscuration of width $4 \%$ of the pupil diameter. The configurations chosen for these diffraction spike patterns were a "plus-sign" four-fold symmetric mask + , or an "asterisk-sign" six-fold symmetric pattern $* .{ }^{18}$ The combined pattern was then given a linear coordinate shear to create elliptical PSF patterns, and the PSF spatial variation for any image

\footnotetext{
18 We use the term "mask" to label such configurations, but we remind the reader that for the $*$ pattern a telescope would only have three struts arranged in a trefoil shape-it is the slit diffraction that results in six spikes in the images.
}

then contained three components, similar to the PSF described in Kitching et al. (2012b: Appendix C, where we refer the reader to figures that show the PSF variation):

1. Static component. This was spatially constant across the image and consisted of (1) a Gaussian smoothing kernel that was added to the PSF size, which had a variance of 0.1 present in all images, and (2) a static additive ellipticity component of 0.05 in $e_{1, \mathrm{PSF}}$ and $e_{2, \mathrm{PSF}}$, to simulate tracking error ( $e_{1}$ and $e_{2}$ are defined in Section 2.3). Details are explained in Kitching et al. (2012b).

2. Deterministic component. This was to simulate the impact of the telescope on the spatially varying PSF size and ellipticity. We used the Jarvis et al. (2008) model with fiducial parameters given in Kitching et al. (2012b) $\left(a_{0}=\right.$ $0.014, a_{1}=0.0005, d_{0}=-0.006, d_{1}=0.001, c_{0}=$ $-0.010)$, which is dominated by primary astigmatism $\left(a_{0}\right)$, primary de-focus $\left(d_{0}\right)$, and coma $\left(c_{0}\right)$.

3. Random component. To simulate the random turbulent effect of the atmosphere we additionally included a random Gaussian field in some images in the ellipticity only, with a Kolmogorov-like power spectrum of $C_{\ell}=\ell^{-11 / 6}$. In fact, subsequent to the formulation of this challenge and launch in 2010, Heymans et al. (2012) found that $C_{\ell} \propto \ell^{-11 / 3}$, the exact power was not known accurately beforehand hence we refer to the $C_{\ell} \propto \ell^{-11 / 6}$ as Kolomogorov-like; this is approximately similar to short exposures from a ground-based observatory for a Moffat PSF, or balloonbased if an Airy PSF is used. We note that the amplitude of the power is also very high, corresponding to exposures of $\simeq 1 \mathrm{~s}$ (see Heymans et al. 2012): we leave an investigation into the impact of varying amplitudes of Kolmogorov power to future work.

The integration of the PSF intensity distribution onto square pixels was achieved by multiplication with a Sinc function in Fourier space (equivalent to convolution with a square boxcar function in real space), followed by sampling at the locations of pixel centers.

\subsection{Data Structure}

The simulation was designed within the constraint that both the download size of the simulation and the upload size of the submissions should be manageable (we limited the download size to $50 \mathrm{~Gb}$ ). Participants were provided with FITS (Wells et al. 1981) images containing "known stars" that were delta functions convolved with a spatially varying PSF. Each star within each image was embedded in a postage stamp of $48 \times$ 48 pixels, and to reduce the size of the images there was no noise in between postage stamps. Participants were then asked to submit a 2D image of the reconstructed PSF at positions in between the known stars; these positions were provided as a catalogue of "asked star" positions. Participants were asked to submit FITS cubes of the reconstructed PSFs (the $x$ and $y$ dimensions representing the $2 \mathrm{D}$ image and the $z$ dimension varying the asked-star positions).

For each image, 1000 asked stars were required. The images were subdivided into 26 sets of 50 images where in each set the properties of the spatial variation, telescope, and static components of the PSF were kept statistically constant, but each had a different realization of any random component, and each also had the asked and known star positions varying. The properties of each set are summarized in Table 1. One aspect to note is that when varying, the size of the PSF in the total flux was 
Table 1

The Properties of Each Set of Images

\begin{tabular}{|c|c|c|c|c|c|c|}
\hline Set & Atmosphere & PSF-Type & Mask & $N_{\text {Stars }}$ & PSF Size (pixels) & Telescope Variation \\
\hline 1 (fid. Airy) & No & Airy & None & 1000 & 3 & None \\
\hline 2 & No & Airy & + & 1000 & 3 & None \\
\hline 3 & No & Airy & $*$ & 1000 & 3 & None \\
\hline 4 & No & Airy & None & 2000 & 3 & None \\
\hline 5 & No & Airy & None & 500 & 3 & None \\
\hline 6 & No & Airy & None & 1000 & 1.5 & None \\
\hline 7 & No & Airy & None & 1000 & 6 & None \\
\hline 8 (fid. Moffat) & No & Moffat & None & 1000 & 3 & None \\
\hline 9 & Yes & Airy & None & 1000 & 3 & None \\
\hline 10 & Yes & Moffat & + & 1000 & 3 & None \\
\hline 11 & Yes & Moffat & $*$ & 1000 & 3 & None \\
\hline 12 & Yes & Moffat & None & 2000 & 3 & None \\
\hline 13 & Yes & Moffat & None & 500 & 3 & None \\
\hline 14 & Yes & Moffat & None & 1000 & 1.5 & None \\
\hline 15 & Yes & Moffat & None & 1000 & 6 & None \\
\hline 16 & No & Airy & None & 1000 & 3 & Astigmatism $a_{0}$ \\
\hline 17 & Yes & Moffat & None & 1000 & 3 & Astigmatism $a_{0}$ \\
\hline 18 & No & Airy & None & 1000 & 3 & De-focus $d_{0}$ \\
\hline 19 & Yes & Moffat & None & 1000 & 3 & De-focus $d_{0}$ \\
\hline 20 & No & Airy & None & 1000 & 3 & Coma $c_{0}$ \\
\hline 21 & Yes & Moffat & None & 1000 & 3 & Coma $c_{0}$ \\
\hline 22 & No & Moffat & + & 1000 & 3 & None \\
\hline 23 & No & Moffat & * & 1000 & 3 & None \\
\hline 24 & No & Moffat & None & 2000 & 3 & None \\
\hline 25 & No & Moffat & None & 500 & 3 & None \\
\hline 26 & No & Moffat & None & 1000 & 1.5 & None \\
\hline
\end{tabular}

Notes. Details are described in Section 2.1. Each category allows a different test: PSF Size allows us to test under-sampling; Atmosphere tests ground-based exposure time dependence; $N_{\text {Stars }}$ tests spatial sampling; Mask tests telescope structure dependence; PSF-Type tests the impact of high spatial frequencies in the PSF profile vs. smooth profiles; Telescope variation allows us to test the impact of three typical distortions found in data. The set order was semi-random so as to prevent participants exploiting any pattern in the set numbering. We label the fiducial sets for the Moffat and Airy profiles.

kept constant for each profile with an integrated signal-to-noise of 100 .

\subsection{Competition Structure}

The competition started in 2010 December and ran for nine months until 2011 September; this was concurrent with the GREAT10 Galaxy Challenge (Kitching et al. 2012a, 2012b). As stated previously the total simulation size was $\sim 50 \mathrm{~Gb}$ and the total size of the uploaded submissions was $\sim 1 \mathrm{~Gb}$ (we allowed participants to tar, zip, or FITS-compress ${ }^{19}$ submissions to reduce size). Data and example code were provided online for participants. ${ }^{20}$

The two parameters of the PSF that most directly impact the ability to interpret observations of galaxies are the ellipticity and the size of the PSF; any residual difference between the ellipticity or size of true PSF, and the respective quantities of the modeled PSF at any particular position, will result in errors and biases in parameters assigned to any galaxy at that position. Weak gravitational lensing is particularly sensitive to these types of error (Massey et al. 2013; Paulin-Henriksson et al. 2008, 2009). The ellipticity and size are defined here using the second order brightness moments of the image as

$$
q_{i j}=\frac{\sum_{p} w_{p} I_{p}\left(\theta_{i}-\bar{\theta}_{i}\right)\left(\theta_{j}-\bar{\theta}_{j}\right)}{\sum_{p} w_{p} I_{p}}, \quad i, j \in\{1,2\},
$$

\footnotetext{
19 http://heasarc.nasa.gov/fitsio/fpack/

$20 \mathrm{http}: / / g r e a t . r o e . a c . u k / d a t a$
}

where the sums are over pixels, $I_{p}$ is the flux in the $p$ th pixel, and $\theta$ is a pixel position $\left(\theta_{1}=x_{p}\right.$ and $\left.\theta_{2}=y_{p}\right)$. In order to make the results regular with regard to the impact of noise but not to constrain the interpretation to compact objects in the postage stamp, we include a weight function $w_{p}$ chosen to be a broad Gaussian with a width of 24 pixels (we leave an investigation of how results vary as a function of weight for future work). These are almost unweighted quadrupole moments in this respect, and as a result, smooth analytical functions may be favored compared to models that try to reproduce details in the wings of the PSF. The weighted ellipticity (or technically the "polarizability") for a PSF in complex notation is defined as

$$
e=\frac{q_{11}-q_{22}+2 \mathrm{i} q_{12}}{q_{11}+q_{22}+2\left(q_{11} q_{22}-q_{12}^{2}\right)^{1 / 2}},
$$

where we have used a definition of ellipticity $|e|=(1-r)(1+$ $r)^{-1}$, where $r$ is the ratio of minor to major axes of the ellipse. For the weighted size we have a similar expression

$$
R^{2}=q_{11}+q_{22}
$$

We can calculate the variance between the ellipticity of the model and true PSF $\sigma^{2}(e) \equiv\left\langle\left(e-e_{\mathrm{PSF}}^{\mathrm{t}}\right)^{2}\right\rangle$ and similarly for the size $\sigma^{2}(R) \equiv\left\langle\left(R-R_{\mathrm{PSF}}^{\mathrm{t}}\right)^{2}\right\rangle$. Submissions were scored in real-time on a leaderboard that displayed the metric $P \equiv$ $\left(1 / 1 / 2\left\langle\sigma^{2}(R)+\sigma^{2}(e)\right\rangle\right)$ where the average was taken over images in each set only (the total number of asked star positions is $N_{\text {Stars }}=N_{\text {images }} \times N_{\text {starsperimage }}$, the average was only taken 


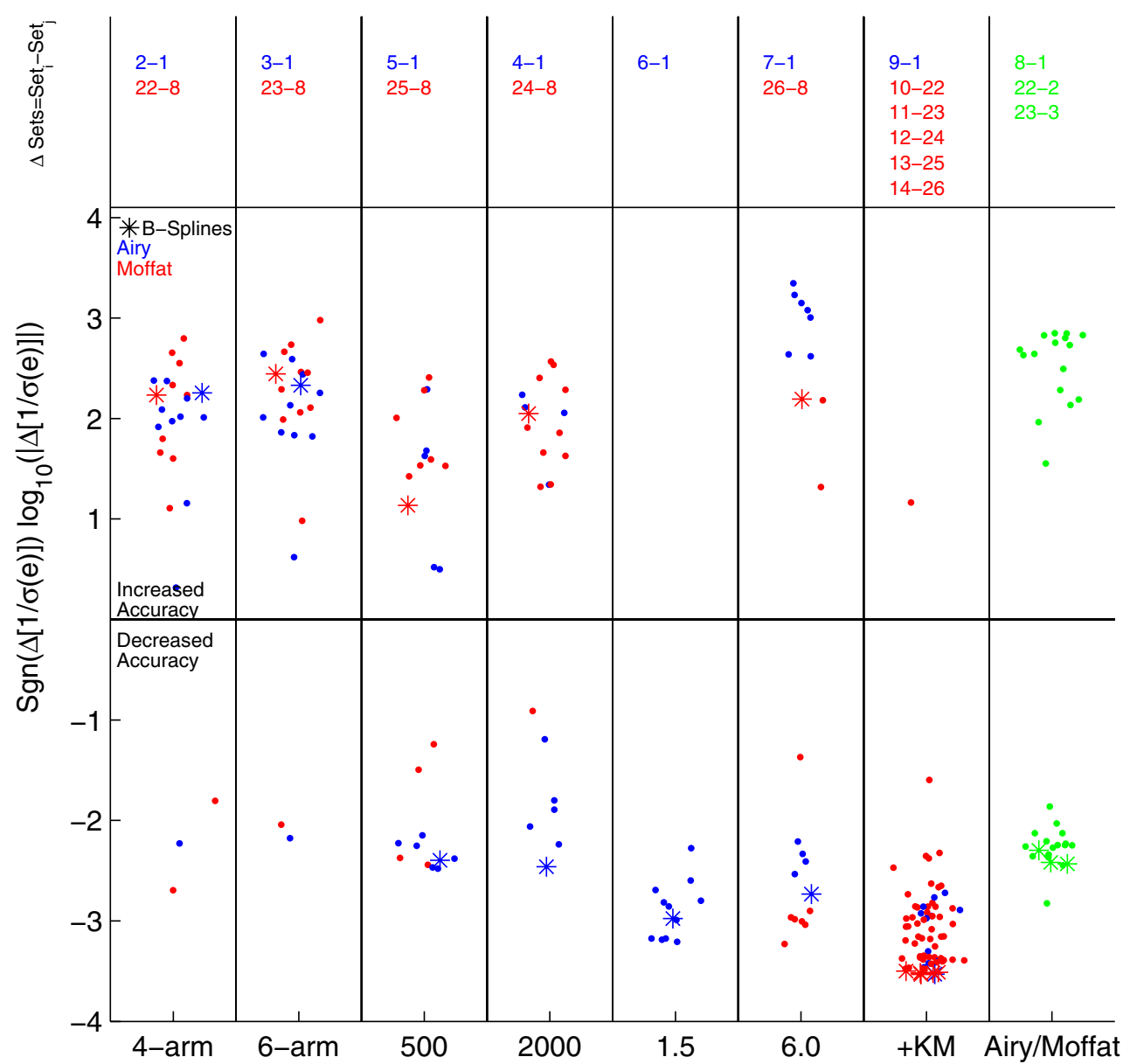

Figure 1. The change in the square root of the inverse variance in the residual ellipticity for each method for each category varied. The sets used in differencing the categories are shown in the upper panels $\left(\operatorname{Set}_{i}-\mathrm{Set}_{j}\right)$, and we refer the reader to Table 1 . Each point represents a method (the stars represent method B-Spline) and points within a bin are randomized within an $x$-bin for clarity. The log of the change is shown, with the sign preserved (i.e., $\operatorname{sgn}[x] \log _{10}[|x|]$ where $\left.x=\left(1 / \sigma(e)_{\text {fiducial }}\right)-(1 / \sigma(e))\right)$ so that negative values represent a decrease in accuracy and positive values an increase in accuracy. The first seven vertical panels show changes for the Moffat (red) and Airy profile (blue); the rightmost panel shows the change in accuracy when the profile is changed from Airy to Moffat but all other aspects of the PSF are kept the same. The parameters varied are the mask (4-arm or 6-arm; changed from no mask), number of stars (500 or 2000 ; changed from 1000), PSF size (1.5 or 6.0 pixels; changed from 3.0 pixels), and the addition of Kolmogorov power in ellipticity.

(A color version of this figure is available in the online journal.)

Table 2

The Results for Ellipticity and Size squared on Set 1 (the Fiducial Airy Set) for Each Method Tested in This Paper

\begin{tabular}{lcccc}
\hline \hline Method Name & $1 / \sigma(e)$ & $\sigma(e) / 10^{-4}$ & $1 /\left[\sigma\left(R^{2}\right) / R^{2}\right]$ & {$\left[\sigma\left(R^{2}\right) / R^{2}\right] / 10^{-3}$} \\
\hline B-Splines & 3953 & 2.53 & 1348 & 0.742 \\
IDW & 3448 & 2.90 & 1212 & 0.825 \\
RBF & 3155 & 3.17 & 1259 & 0.794 \\
RBF-thin & 2985 & 3.35 & 1258 & 0.795 \\
Kriging & 1049 & 9.53 & 490 & 2.042 \\
Gaussianlets & 1473 & 6.79 & 392 & 2.548 \\
IDW Stk & 1058 & 9.45 & 277 & 3.604 \\
PSFEx & 1279 & 7.82 & 378 & 2.647 \\
Shapelets & 1256 & 7.96 & 379 & 2.642 \\
PCA+Kriging & 1339 & 7.47 & 314 & 3.180 \\
MoffatGP & 2545 & 3.93 & 429 & 2.331 \\
Stacking & 1441 & 6.94 & 309 & 3.237
\end{tabular}

over $N_{\text {images }}$ ), such that that a mean variance of $10^{-3}$ in both ellipticity and size would have $P \sim 1.0$.

The $P$ metric, while indicatively ranking the methods, does not offer any insight into the performance of a method on ellipticity and size reconstruction. In this paper we will present
Table 3

The Results for Ellipticity and Size squared on Set 8 (the Fiducial Moffat Set) for Each Method Tested in This Paper

\begin{tabular}{lcccc}
\hline \hline Method Name & $1 / \sigma(e)$ & $\sigma(e) / 10^{-4}$ & $1 /\left[\sigma\left(R^{2}\right) / R^{2}\right]$ & {$\left[\sigma\left(R^{2}\right) / R^{2}\right] / 10^{-3}$} \\
\hline B-Splines & 3690 & 2.71 & 1406 & 0.711 \\
IDW & 3215 & 3.11 & 1309 & 0.764 \\
RBF & 2967 & 3.37 & 1167 & 0.857 \\
RBF-thin & 2809 & 3.56 & 1163 & 0.860 \\
Kriging & 1477 & 6.77 & 645 & 1.551 \\
Gaussianlets & 2041 & 4.90 & 476 & 2.099 \\
IDW Stk & 1250 & 8 & 362 & 2.759 \\
PSFEx & 610 & 16.40 & 296 & 3.374 \\
Shapelets & 1931 & 5.18 & 696 & 1.436 \\
PCA+Kriging & 1161 & 8.61 & 351 & 2.853 \\
MoffatGP & 2857 & 3.50 & 139 & 7.209 \\
Stacking & 1259 & 7.94 & 309 & 3.236
\end{tabular}

quantities that relate to the principal properties of the PSF more directly. These are the standard deviation of the mean of the residuals of the ellipticity $\sigma(e)$ and size squared $\sigma\left(R^{2}\right) / R^{2}$ over all asked stars, i.e., we compute the error on the mean of the residuals (the sample variance computed using centered 


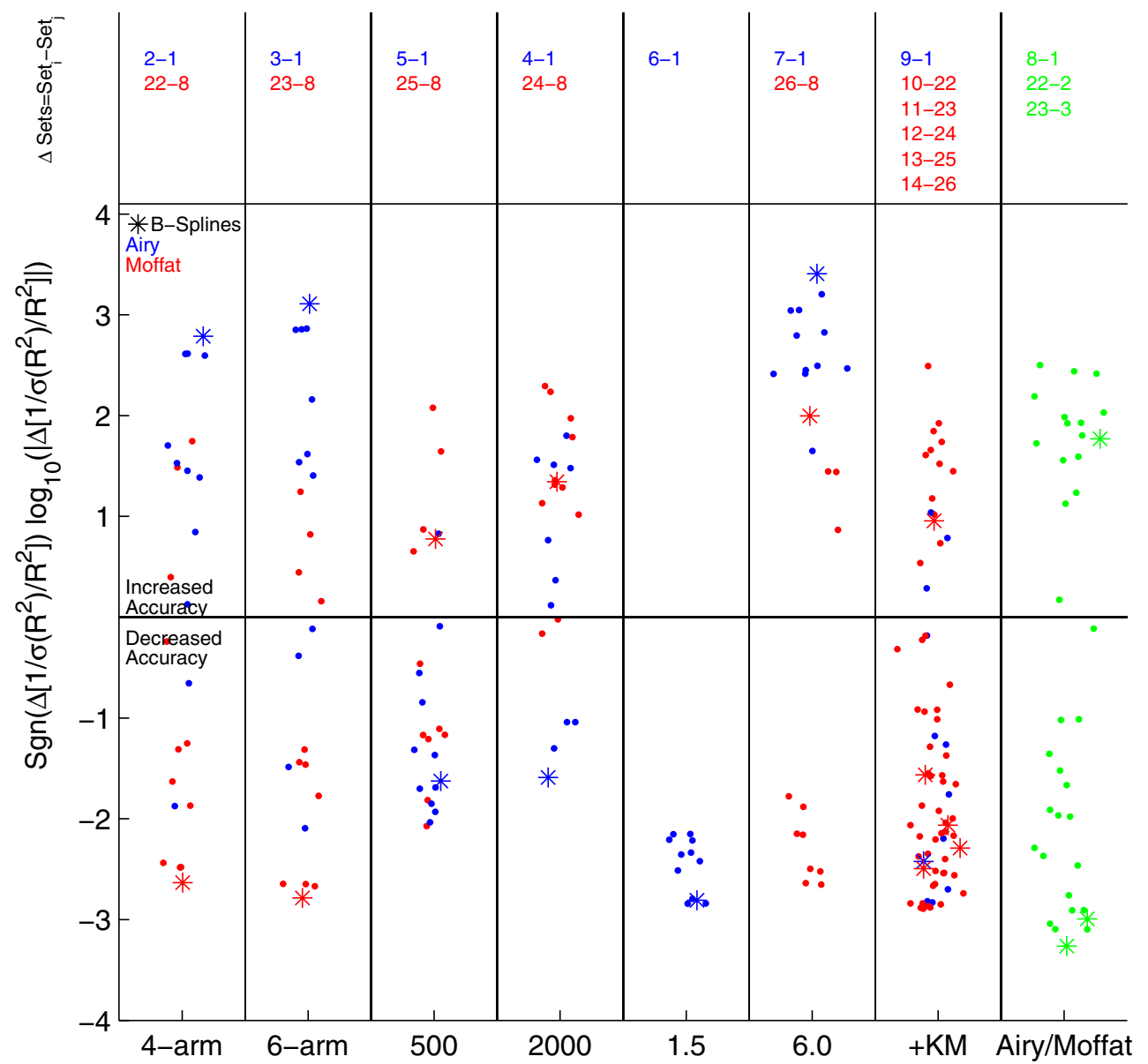

Figure 2. The change in the square root of the inverse variance in the residual size squared for each method for each category varied. The sets used in differencing the categories are shown in the upper panels $\left(\operatorname{Set}_{i}-\mathrm{Set}_{j}\right)$, and we refer the reader to Table 1. Each point represents a method, the stars represent method B-Spline, points within a bin are randomized in within an $x$-bin for clarity. The log of the change is shown, with the sign preserved (i.e., $\operatorname{sgn}[x] \log _{10}[|x|]$ where $\left.x=\left(1 /\left[\sigma\left(R^{2}\right) / R^{2}\right]_{\text {fiducial }}\right)-\left(1 /\left[\sigma\left(R^{2}\right) / R^{2}\right]\right)\right)$ so that negative values represent a decrease in accuracy and positive values an increase in accuracy. The first seven vertical panels show changes for the Moffat (red) and Airy profile (blue), the rightmost panel shows the change in accuracy when the profile is changed from Airy to Moffat but all other aspects of the PSF at kept the same. The parameters varied are the mask (4-arm or 6-arm; changed from no mask), number of stars (500 or 2000; changed from 1000), PSF size (1.5 or 6.0 pixels; changed from 3.0 pixels) and the addition of Kolmogorov power in ellipticity.

(A color version of this figure is available in the online journal.)

second order moments). We assume that any mean bias could be removed through cross-validation, in this sense it is a generous analysis to those methods with a mean residual. We average these quantities over the 50 images in each set, but in fact for all methods we find that the fractional error between images in a set is $\lesssim 10 \%$.

\section{RESULTS}

In total, 30 submissions were made from 9 teams. As a baseline benchmark, a method in which all stars were simply stacked in an image was created, where no spatial variation in the reconstructed stars was present. Several methods generated low scores due to misunderstanding of simulation details, resulting in scores below the benchmark, and in this paper we summarize only those not affected by these issues. In the following we choose the best performing submission, for size, for each of the 12 distinct method entries. All of the submitted methods are described in the Appendix. We show the results on the fiducial Airy set (set 1 in Table 1) and the fiducial Moffat set (set 8 in Table 1) in Tables 2 and 3, respectively. In Figures 1 and 2 we present general behaviors of methods over the sets as categories were changed, but for a quantitative presentation of each method we refer the reader to Figures 3-7 where we show pictographic tables of results. In the square root of the inverse variance, and referring to Figures 1 and 2, for example, the error between images in a set of $10 \%$ results in changes of $\Delta[1 / \sigma(e)] \approx \pm 200$ and similarly for $R^{2}$, not being significant for most methods individually, but can be significant collectively.

Overall we find that the B-Splines, Inverse Distance Weighting (IDW), and Radial Basis Function (RBF) methods reconstruct the ellipticity and size most accurately (see Gentile et al. 2013), with $\sigma(e) \approx 2.5 \times 10^{-4}$ and $\sigma\left(R^{2}\right) / R^{2} \approx 7.4 \times 10^{-4}$ over all sets. ${ }^{21}$ We note, however, that this is a snapshot of performance and that further investigations into tunable aspects of code could result in improvements in all methods.

We summarize the behavior of the submissions below. In each test all other parameters are kept fixed except those discussed (with fiducial values of 1000 known star positions, no mask, and telescope parameters given in Section 2). We refer to Figures 1 and 2 which show the change in the square root of the inverse variance of the reconstructed PSFs over the fiducial sets (set 1 for Airy and set 8 for Moffat profiles; see Table 2) when each of the categories is varied. In Figures 3-7 we show pictographic tables of results.

\footnotetext{
${ }^{21}$ B-Splines also achieved the highest leaderboard $P$ value.
} 


\begin{tabular}{|c|c|c|c|c|}
\hline $1 / \sigma(e)$ & $\begin{array}{c}\bigcirc \\
10^{4}\end{array}$ & $\begin{array}{c}\circ \\
10^{3}\end{array}$ & $10^{2}$ & $\begin{array}{l}\text { Moffat+KM } \\
\text { Airy } \\
\text { Moffat }\end{array}$ \\
\hline B-Splines & 833953 & & $\begin{array}{l}561 \\
4112 \\
3861\end{array}$ & $\begin{array}{l}557 \\
4167 \\
3968\end{array}$ \\
\hline IDW & $\begin{array}{r}3448 \\
3215 \\
\end{array}$ & & $\begin{array}{l}855 \\
3551 \\
3571 \\
\end{array}$ & $\begin{array}{l}8525 \\
35676 \\
3676\end{array}$ \\
\hline RBF & $\begin{array}{r}3155 \\
2967 \\
\end{array}$ & & $\begin{array}{r}506 \\
8357 \\
3030 \\
\end{array}$ & $\begin{array}{l}507 \\
8257 \\
3096 \\
3096\end{array}$ \\
\hline RBF-thin & 822985 & & $\begin{array}{r}889 \\
82067 \\
2849\end{array}$ & $\begin{array}{l}891 \\
83058 \\
2924\end{array}$ \\
\hline Kriging & $: \begin{array}{l}1049 \\
1477\end{array}$ & & $\begin{array}{r}: 555 \\
: 1208 \\
1692 \\
\end{array}$ & $\begin{array}{r}: 555 \\
: 1325 \\
1764 \\
\end{array}$ \\
\hline Gaussianlets & $\begin{array}{r}1473 \\
2041\end{array}$ & & $\begin{array}{l}\quad 532 \\
8157 \\
2212\end{array}$ & $\begin{array}{l}: 534 \\
81653 \\
2331 \\
2331\end{array}$ \\
\hline IDW Stk & $\begin{array}{l}1058 \\
: 1250 \\
\end{array}$ & & $\begin{array}{r}\quad 544 \\
: 889 \\
\end{array}$ & $\begin{array}{l}: 546 \\
: 908 \\
1445 \\
\end{array}$ \\
\hline PSFEx & $\begin{array}{l}: 1279 \\
610\end{array}$ & & $\begin{array}{l}: \begin{array}{l}415 \\
1515 \\
1236\end{array} \\
\end{array}$ & $\begin{array}{l}: 487 \\
: 1669 \\
1562\end{array}$ \\
\hline Shapelets & $: \begin{array}{l}1256 \\
1931\end{array}$ & & $\begin{array}{r}\quad 540 \\
: 1271 \\
1976 \\
\end{array}$ & $\begin{array}{r}\begin{array}{r}540 \\
1325 \\
2028 \\
202\end{array} \\
\end{array}$ \\
\hline PCA+Kriging & $\begin{array}{l}1339 \\
1161 \\
\end{array}$ & & $\begin{array}{r}553 \\
81577 \\
1613 \\
\end{array}$ & $\begin{array}{r}515 \\
8179 \\
1706 \\
536\end{array}$ \\
\hline MoffatGP & $\begin{array}{r}2545 \\
2857 \\
\end{array}$ & & $\begin{array}{r}5441 \\
82639 \\
\end{array}$ & $\begin{array}{r}836 \\
82611 \\
2747 \\
547\end{array}$ \\
\hline Stacking & $\begin{array}{r}1441 \\
1259 \\
\end{array}$ & & 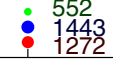 & 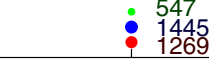 \\
\hline
\end{tabular}

\begin{tabular}{|c|c|c|c|c|}
\hline $1 /\left[\sigma\left(R^{2}\right) / R^{2}\right]$ & $\begin{array}{c}\bigcirc \\
10^{4}\end{array}$ & $\begin{array}{c}\circ \\
10^{3}\end{array}$ & $10^{2}$ & $\begin{array}{l}\text { Moffat+KM } \\
\text { Airy } \\
\text { Moffat }\end{array}$ \\
\hline B-Splines & $: \begin{array}{l}1348 \\
1406\end{array}$ & & 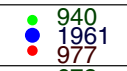 & \begin{tabular}{r}
807 \\
\hdashline$\quad 2632$ \\
798 \\
\end{tabular} \\
\hline IDW & $\begin{array}{l}1212 \\
1309 \\
\end{array}$ & & $\begin{array}{l}: 672 \\
: 1608 \\
1035 \\
\end{array}$ & \begin{tabular}{r}
$\because 621$ \\
\hdashline$\quad 1942$ \\
843 \\
\end{tabular} \\
\hline RBF & $: 1259$ & & $\begin{array}{l}418 \\
: 1672 \\
863\end{array}$ & \begin{tabular}{l}
$\because 393$ \\
\hdashline 1976 \\
\hdashline 723
\end{tabular} \\
\hline RBF-thin & $\begin{array}{l}1258 \\
1163 \\
\end{array}$ & & $\begin{array}{l}401 \\
: \begin{array}{l}4667 \\
861\end{array} \\
\end{array}$ & $\begin{array}{r}375 \\
: 1969 \\
722 \\
\end{array}$ \\
\hline Kriging & $: \begin{array}{l}490 \\
645 \\
\end{array}$ & & $\begin{array}{l}\vdots \begin{array}{r}500 \\
540 \\
647\end{array} \\
\end{array}$ & $\begin{array}{r}\vdots 490 \\
: 635 \\
624 \\
\end{array}$ \\
\hline Gaussianlets & $: \begin{array}{l}392 \\
476 \\
\end{array}$ & & $\begin{array}{r}\quad 357 \\
: 417 \\
\end{array}$ & $\begin{array}{r}: 338 \\
: 434 \\
447 \\
\end{array}$ \\
\hline IDW Stk & $: \begin{array}{l}277 \\
362 \\
\end{array}$ & & $\begin{array}{l}\vdots 344 \\
\vdots 311 \\
289 \\
\end{array}$ & $\begin{array}{r}\vdots 342 \\
\vdots 312 \\
365 \\
\end{array}$ \\
\hline PSFEx & $\begin{array}{l}378 \\
296 \\
\end{array}$ & & $\begin{array}{r}167 \\
: 373 \\
327 \\
\end{array}$ & $\begin{array}{r}175 \\
: \quad 347 \\
314 \\
\end{array}$ \\
\hline Shapelets & $\begin{array}{r}379 \\
: \quad 696 \\
\end{array}$ & & $\begin{array}{l}\vdots 618 \\
: 378 \\
654 \\
\end{array}$ & $\begin{array}{r}600 \\
: 376 \\
637 \\
\end{array}$ \\
\hline PCA+Kriging & $: \begin{array}{l}314 \\
351 \\
\end{array}$ & & $\begin{array}{r}: 476 \\
\vdots 343 \\
406 \\
\end{array}$ & $\begin{array}{l}: \begin{array}{l}441 \\
340 \\
357\end{array} \\
\end{array}$ \\
\hline MoffatGP & $\begin{array}{l}429 \\
: \quad 139 \\
\end{array}$ & & 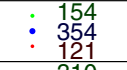 & 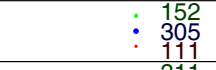 \\
\hline Stacking & $\begin{array}{r}309 \\
\vdots \quad 309 \\
\end{array}$ & & $\begin{array}{r}\quad 310 \\
310 \\
310 \\
\end{array}$ & $\begin{array}{r}\vdots 31 \\
310 \\
\vdots \\
310 \\
\end{array}$ \\
\hline
\end{tabular}

Figure 3. The square root of the inverse variance in the residual ellipticity and size squared for each method (horizontal panels) for the three mask cases (no mask, 4-arm + and 6-arm $*$ ) for the Moffat-plus-Kolmogorov case (green), Moffat with no Kolmogorov (red), and the Airy (blue) profile. The circles represent the square root of the inverse variance of the residual ellipticity and size squared where the area scales in proportion to these parameters and the numbers are given next to each circle; a key is given in the top panel. Where no number/circle is provided there was no set for this combination of PSF type and mask type. Fractional errors on the square root of the inverse variances are $\approx 10 \%$ for all methods.

(A color version of this figure is available in the online journal.)

\begin{tabular}{|c|c|c|c|c|}
\hline $1 / \sigma(e)$ & $\begin{array}{l}\bigcirc \\
10^{4}\end{array}$ & $\begin{array}{c}\circ \\
10^{3}\end{array}$ & $10^{2}$ & $\begin{array}{l}\text { Moffat+KM } \\
\text { Airy } \\
\text { Moffat }\end{array}$ \\
\hline B-Splines & $\begin{array}{l}8538 \\
8584 \\
3704\end{array}$ & & 83953 & $\begin{array}{l}560 \\
3663 \\
3802\end{array}$ \\
\hline IDW & $\begin{array}{l}5 \\
5 \\
35255 \\
3472\end{array}$ & & 83448 & $\begin{array}{l}601 \\
3448 \\
3584\end{array}$ \\
\hline RBF & $\begin{array}{l}4488 \\
82950 \\
2950\end{array}$ & & 83155 & $\begin{array}{l}537 \\
83040 \\
3040 \\
\end{array}$ \\
\hline RBF-thin & $\begin{array}{l}44617 \\
82878 \\
2778\end{array}$ & & $8 \begin{array}{l}2985 \\
2809\end{array}$ & $\begin{array}{r}520 \\
82907 \\
2890 \\
\end{array}$ \\
\hline Kriging & $\begin{array}{l}528 \\
: 1053 \\
1504\end{array}$ & & $\begin{array}{r}1049 \\
: \quad 1477 \\
\end{array}$ & $\begin{array}{r}578 \\
: \quad 1049 \\
: \quad 499 \\
\end{array}$ \\
\hline Gaussianlets & $\begin{array}{r}5119 \\
8 \\
2075\end{array}$ & & $\begin{array}{l}1473 \\
2041\end{array}$ & $\begin{array}{l}567 \\
81495 \\
2083\end{array}$ \\
\hline IDW Stk & $\begin{array}{l}: 55406 \\
: 1441 \\
\end{array}$ & & $\begin{array}{r}1058 \\
1250 \\
\end{array}$ & 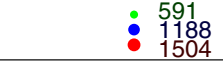 \\
\hline PSFEx & $\begin{array}{l}135 \\
: 3748 \\
374\end{array}$ & & $: \begin{array}{l}1279 \\
610\end{array}$ & $\begin{array}{l}261 \\
: \begin{array}{l}26133 \\
803\end{array} \\
\end{array}$ \\
\hline Shapelets & $\begin{array}{r}5355 \\
81259 \\
1965 \\
\end{array}$ & & $\begin{array}{r}1256 \\
1931 \\
\end{array}$ & $\begin{array}{r}541 \\
: 1241 \\
1976 \\
\end{array}$ \\
\hline PCA+Kriging & $\begin{array}{l}4586 \\
: 1036 \\
884\end{array}$ & & $\begin{array}{r}1339 \\
1161 \\
\end{array}$ & $\begin{array}{r}558 \\
: \quad 1511 \\
1504 \\
\end{array}$ \\
\hline MoffatGP & $\begin{array}{r}5390 \\
82740 \\
2959\end{array}$ & & 82545 & $\begin{array}{r}541 \\
8 \\
8 \\
2849 \\
\end{array}$ \\
\hline Stacking & $\begin{array}{r}: 5480 \\
: 1300 \\
1299 \\
\end{array}$ & & $\begin{array}{l}1441 \\
1259 \\
\end{array}$ & \begin{tabular}{r}
547 \\
$: 1267$ \\
\hdashline$\quad 1280$ \\
\end{tabular} \\
\hline & 500 & & 1000 & 2000 \\
\hline
\end{tabular}

\begin{tabular}{|c|c|c|c|c|}
\hline $1 /\left[\sigma\left(R^{2}\right) / R^{2}\right]$ & $\underset{10^{4}}{\bigcirc}$ & $\begin{array}{c}\circ \\
10^{3}\end{array}$ & $10^{2}$ & $\begin{array}{l}\text { Moffat+KM } \\
\text { Airy } \\
\text { Moffat }\end{array}$ \\
\hline B-Splines & $\begin{array}{l}1233 \\
: 355 \\
1412\end{array}$ & & $: \begin{array}{l}1348 \\
1406\end{array}$ & $\begin{array}{l}1297 \\
: \begin{array}{l}1309 \\
1429\end{array}\end{array}$ \\
\hline IDW & $\begin{array}{l}7764 \\
: 1429 \\
\end{array}$ & & $: \begin{array}{l}1212 \\
1309 \\
\end{array}$ & $\begin{array}{l}737 \\
: \begin{array}{l}737 \\
1242 \\
1481\end{array} \\
\end{array}$ \\
\hline RBF & $\begin{array}{l}4501 \\
: \begin{array}{l}4211 \\
152\end{array} \\
\end{array}$ & & $: \begin{array}{r}1259 \\
1167 \\
\end{array}$ & $\begin{array}{l}463 \\
\vdots 248 \\
1866 \\
\end{array}$ \\
\hline RBF-thin & $\begin{array}{l}426 \\
: \begin{array}{l}1208 \\
1148\end{array} \\
\end{array}$ & & $\begin{array}{r}1258 \\
: 1163 \\
\end{array}$ & $\begin{array}{c}446 \\
: \begin{array}{l}4447 \\
1183\end{array} \\
\end{array}$ \\
\hline Kriging & $\begin{aligned} & 518 \\
&: 486 \\
& 629 \\
&\end{aligned}$ & & $: \begin{array}{l}490 \\
645 \\
\end{array}$ & 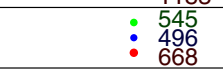 \\
\hline Gaussianlets & $\begin{array}{r}\quad 365 \\
: 361 \\
\end{array}$ & & $: \begin{array}{r}392 \\
476 \\
\end{array}$ & $\begin{array}{r}390 \\
: \quad 395 \\
\end{array} 490$ \\
\hline IDW Stk & $\begin{array}{l}173 \\
: \quad 254 \\
407\end{array}$ & & $: \begin{array}{l}277 \\
362\end{array}$ & $\begin{array}{l}396 \\
: \begin{array}{l}314 \\
424\end{array}\end{array}$ \\
\hline PSFEx & $\begin{array}{l}: \begin{array}{l}466 \\
: 36 \\
178\end{array} \\
\end{array}$ & & $: \begin{array}{l}378 \\
296\end{array}$ & $\begin{array}{r}159 \\
: \begin{array}{r}410 \\
391\end{array} \\
\end{array}$ \\
\hline Shapelets & 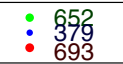 & & $\begin{array}{l}379 \\
: \quad 696 \\
\end{array}$ & $\begin{array}{r}\quad 648 \\
\vdots 379 \\
695 \\
\end{array}$ \\
\hline PCA+Kriging & 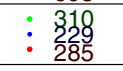 & & $\begin{array}{r}314 \\
: 351 \\
\end{array}$ & 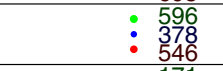 \\
\hline MoffatGP & $\begin{array}{r}: 184 \\
: \quad 488 \\
143\end{array}$ & & $\begin{array}{r}\quad 429 \\
: \quad 139 \\
\end{array}$ & $\begin{array}{r}171 \\
: \quad 409 \\
139 \\
\end{array}$ \\
\hline Stacking & $\begin{array}{r}311 \\
\vdots \\
316 \\
\end{array}$ & & $\begin{array}{r}309 \\
: 309 \\
\end{array}$ & $\begin{array}{r}308 \\
\vdots \quad 308 \\
\vdots \quad 308 \\
\end{array}$ \\
\hline & 500 & & 1000 & 2000 \\
\hline
\end{tabular}

Figure 4. The square root of the inverse variance in the residual ellipticity and size squared for each method (horizontal panels) for the three known-star number cases (500, 1000, 2000 stars) for the Moffat-plus-Kolmogorov case (green), Moffat with no Kolmogorov (red), and the Airy (blue) profile. The circles represent the square root of the inverse variance of the residual ellipticity and size squared where the area scales in proportion to these parameters and the numbers are given next to each circle; a key is given in the top panel. Where no number/circle is provided there was no set for this combination of PSF type and number of stars. Fractional errors on the square root of the inverse variances are $\approx 10 \%$ for all methods.

(A color version of this figure is available in the online journal.) 


\begin{tabular}{|c|c|c|c|c|}
\hline $1 / \sigma(e)$ & $\begin{array}{c}\bigcirc \\
10^{4}\end{array}$ & $\begin{array}{c}\circ \\
10^{3}\end{array}$ & $\begin{array}{c}\cdot \\
10^{2}\end{array}$ & $\begin{array}{l}\text { Moffat+KM } \\
\text { Airy } \\
\text { Moffat }\end{array}$ \\
\hline B-Splines & $\therefore \quad 5003$ & & $\begin{array}{r}3953 \\
3846\end{array}$ & $\begin{array}{l}8_{3413}^{559} 3490 \\
3690 \\
\end{array}$ \\
\hline IDW & $\begin{array}{l}5682 \\
-\quad 1912\end{array}$ & & 83448 & $\begin{aligned} 583 \\
83106 \\
3215\end{aligned}$ \\
\hline RBF & $\begin{array}{l}506 \\
\quad 1661\end{array}$ & & $8 \begin{array}{l}3155 \\
2967\end{array}$ & $\begin{array}{r}531 \\
8289 \\
2967 \\
\end{array}$ \\
\hline RBF-thin & $: 4988$ & & 82985 & $\begin{array}{r}515 \\
8270 \\
2809\end{array}$ \\
\hline Kriging & $: 454$ & & $\begin{array}{l}1049 \\
\end{array}$ & $\begin{array}{r}576 \\
82747 \\
81477 \\
\end{array}$ \\
\hline Gaussianlets & $: 4886$ & & $\begin{array}{l}1473 \\
: 949 \\
\end{array}$ & $\begin{array}{r}536 \\
8690 \\
2041 \\
2040\end{array}$ \\
\hline IDW Stk & $: 281$ & & $:{ }_{330}^{1058}$ & $\begin{array}{l}: 583 \\
: \begin{array}{l}5493 \\
1250\end{array}\end{array}$ \\
\hline PSFEx & $: 468$ & & $: \begin{array}{l}1279 \\
762\end{array}$ & $\begin{array}{r}493 \\
: \quad 1695 \\
610\end{array}$ \\
\hline Shapelets & $: 517$ & & $: \begin{array}{l}1256 \\
965\end{array}$ & $\begin{array}{r}544 \\
82268 \\
81931 \\
\end{array}$ \\
\hline PCA+Kriging & $: \quad 164$ & & $\begin{array}{l}1339 \\
149 \\
\end{array}$ & $\begin{array}{r}576 \\
82747 \\
\end{array}$ \\
\hline MoffatGP & : 5224 & & $8 \begin{array}{l}2545 \\
164\end{array}$ & $\begin{array}{r}547 \\
8375 \\
2857 \\
\end{array}$ \\
\hline Stacking & $\begin{array}{r}542 \\
: \quad 1252 \\
\end{array}$ & & $\begin{array}{l}1441 \\
1259 \\
\end{array}$ & $\begin{array}{r}546 \\
: \quad 1279 \\
1 \\
1259\end{array}$ \\
\hline
\end{tabular}

\begin{tabular}{|c|c|c|c|}
\hline $1 /\left[\sigma\left(R^{2}\right) / R^{2}\right]$ & 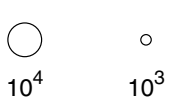 & $10^{2}$ & $\begin{array}{l}\text { Moffat+KM } \\
\text { Airy } \\
\text { Moffat }\end{array}$ \\
\hline B-Splines & $: 1195$ & $: \begin{array}{l}1348 \\
1506\end{array}$ & $\begin{array}{l}886 \\
3906 \\
1406\end{array}$ \\
\hline IDW & $: 5884$ & $: \begin{array}{r}1212 \\
1233 \\
\end{array}$ & $\begin{array}{r}711 \\
8 \quad 209 \\
1309 \\
\end{array}$ \\
\hline RBF & $: 565$ & 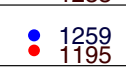 & \begin{tabular}{l}
452 \\
\hdashline $\begin{array}{l}2375 \\
1167\end{array}$ \\
\end{tabular} \\
\hline RBF-thin & $: 412$ & $: \begin{array}{l}1258 \\
190\end{array}$ & $\begin{array}{l}442 \\
8364 \\
1163\end{array}$ \\
\hline Kriging & : 222 & $\begin{array}{r}490 \\
: \quad 210 \\
\end{array}$ & $\begin{array}{r}1098 \\
: 1112 \\
645 \\
\end{array}$ \\
\hline Gaussianlets & : 295 & $: \begin{array}{r}392 \\
333 \\
\end{array}$ & $\begin{array}{l}\mathbf{4} 480 \\
652 \\
476 \\
\end{array}$ \\
\hline IDW Stk & $\therefore 59$ & : 277 & $\begin{array}{l}\quad 508 \\
: \begin{array}{l}539 \\
362\end{array}\end{array}$ \\
\hline PSFEx & $: 2289$ & $: \begin{array}{l}378 \\
236\end{array}$ & 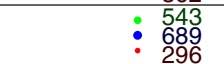 \\
\hline Shapelets & $: 244$ & : 379 & $\begin{array}{l}: 1079 \\
: 672 \\
696\end{array}$ \\
\hline PCA+Kriging & .24 & \begin{tabular}{r}
314 \\
\hdashline$\quad 18$ \\
\end{tabular} & $\begin{array}{l}1479 \\
: \begin{array}{l}143 \\
351\end{array} \\
\end{array}$ \\
\hline MoffatGP & $: 161$ & $\begin{array}{l}429 \\
: \quad 146 \\
\end{array}$ & \begin{tabular}{r}
433 \\
\hdashline$\quad 474$ \\
139 \\
\end{tabular} \\
\hline Stacking & $: 169$ & $\begin{array}{r}309 \\
\vdots \quad 168 \\
\end{array}$ & \begin{tabular}{r}
594 \\
$: 592$ \\
\hdashline$\quad 309$ \\
\end{tabular} \\
\hline
\end{tabular}

Figure 5. The square root of the inverse variance in the residual ellipticity and size squared for each method (horizontal panels) for the three PSF size cases (1.5, 3.0 , and 6.0 pixels) for the Moffat-plus-Kolmogorov case (green), Moffat with no Kolmogorov (red), and the Airy (blue) profile. The circles represent the square root of the inverse variance of the residual ellipticity and size squared where the area scales in proportion to these parameters and the numbers are given next to each circle; a key is given in the top panel. Where no number/circle is provided there was no set for this combination of PSF type and PSF size. Fractional errors on the square root of the inverse variances are $\approx 10 \%$ for all methods.

(A color version of this figure is available in the online journal.)

\begin{tabular}{|c|c|c|c|}
\hline $1 / \sigma(e)$ & $\begin{array}{c}\bigcirc \\
10^{4}\end{array}$ & $\begin{array}{c}\circ \\
10^{3}\end{array}$ & $10^{2}$ \\
\hline B-Splines & 3953 & & - 562 \\
\hline IDW & - 3448 & & - 547 \\
\hline RBF & - 3155 & & - 508 \\
\hline RBF-thin & - 2985 & & - 491 \\
\hline Kriging & - 1049 & & - 524 \\
\hline Gaussianlets & - 1473 & & - 533 \\
\hline IDW Stk & - 1058 & & - 476 \\
\hline PSFEx & - 1279 & & - 436 \\
\hline Shapelets & - 1256 & & - 535 \\
\hline $\mathrm{PCA}+$ Kriging & - 1339 & & - 562 \\
\hline MoffatGP & - 2545 & & - 536 \\
\hline Stacking & - 1441 & & - 551 \\
\hline
\end{tabular}

\begin{tabular}{|c|c|c|c|c|}
\hline $1 /\left[\sigma\left(R^{2}\right) / R^{2}\right]$ & $\begin{array}{l}\underset{10^{4}}{O} \\
\end{array}$ & $\begin{array}{c}\circ \\
10^{3}\end{array}$ & $10^{2}$ & \\
\hline B-Splines & - 1348 & & & 1083 \\
\hline IDW & - 1212 & & & 712 \\
\hline RBF & - 1259 & & & 603 \\
\hline RBF-thin & - 1258 & & & 584 \\
\hline Kriging & - 490 & & & 475 \\
\hline Gaussianlets & - 392 & & & 374 \\
\hline IDW Stk & - 277 & & & 279 \\
\hline PSFEx & - 378 & & & 221 \\
\hline Shapelets & - 379 & & & 385 \\
\hline $\mathrm{PCA}+$ Kriging & - 314 & & & 325 \\
\hline MoffatGP & - 429 & & & 372 \\
\hline Stacking & - 309 & & - & 307 \\
\hline
\end{tabular}

Figure 6. The square root of the inverse variance in the residual ellipticity and size squared for each method (horizontal panels) for the two cases where a Kolmogorov power is added, or not to a set with an Airy (blue) profile. The circles represent the square root of the inverse variance of the residual ellipticity and size squared where the area scales in proportion to these parameters and the numbers are given next to each circle; a key is given in the top panel. Fractional errors on the square root of the inverse variances are $\approx 10 \%$ for all methods.

(A color version of this figure is available in the online journal.) 


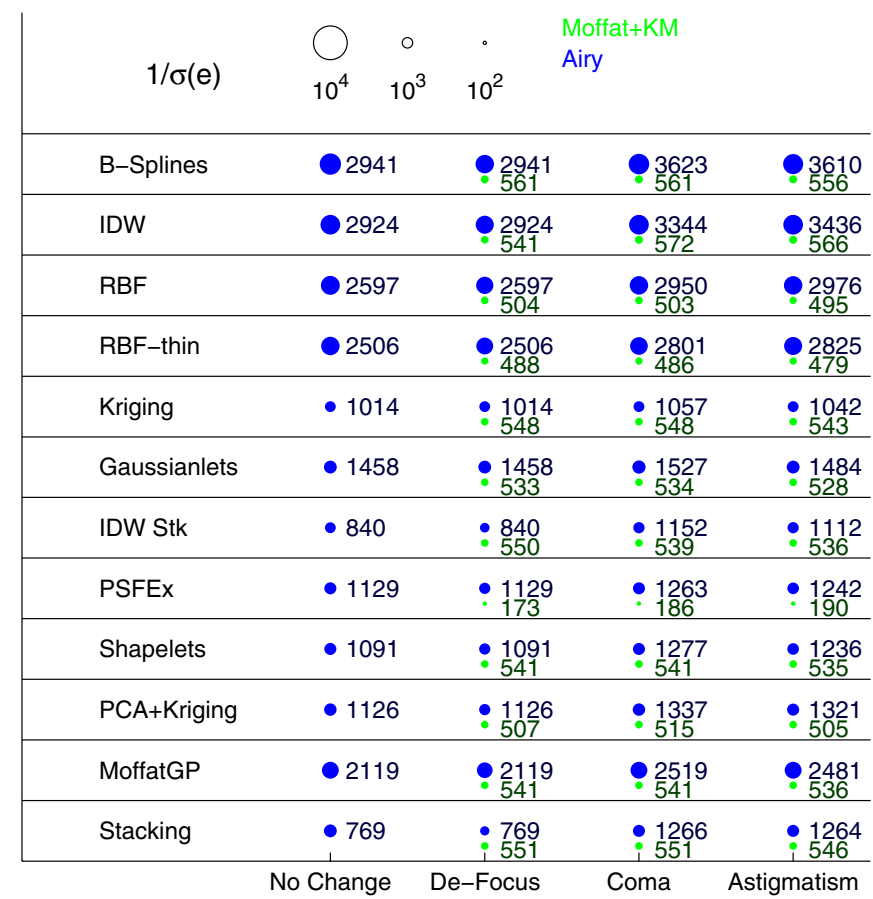

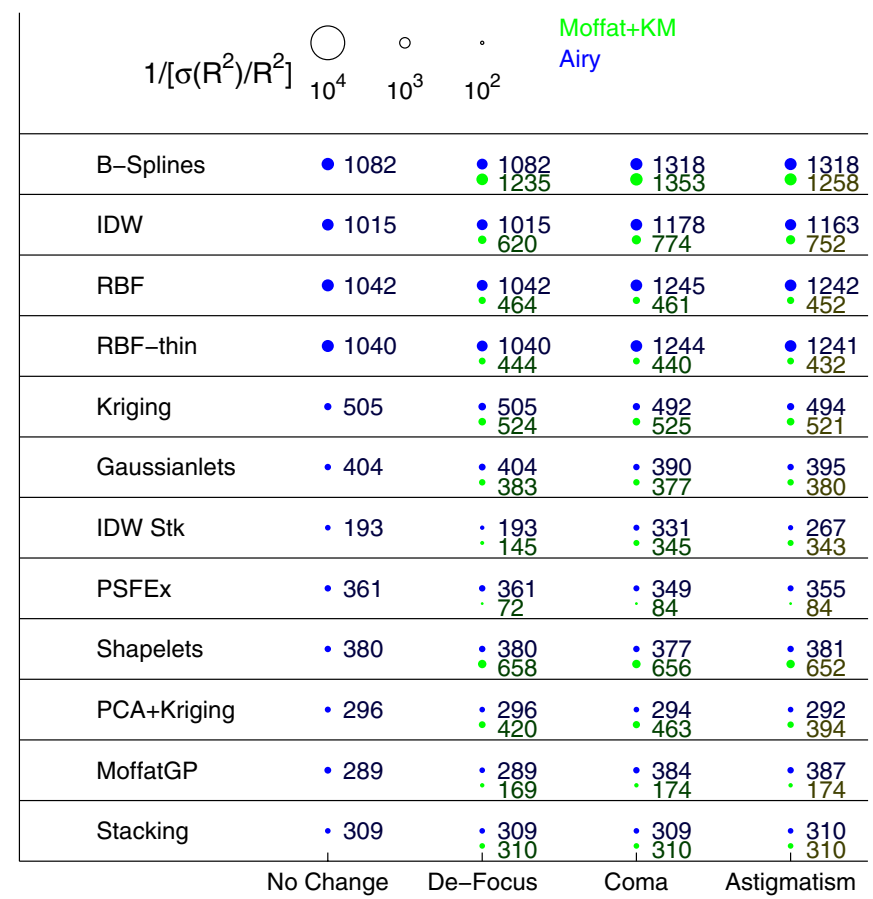

Figure 7. The square root of the inverse variance in the residual ellipticity and size squared for each method (horizontal panels) for the cases where the telescope parameters are varied for the Airy (blue) profile and the Moffat-plus-Kolmogorov profile (green). The circles represent the square root of the inverse variance of the residual ellipticity and size squared where the area scales in proportion to these parameters and the numbers are given next to each circle; a key is given in the top panel. Where no number/circle is provided there was no set for this combination of PSF type and telescope parameter. Fractional errors on the square root of the inverse variances are $\approx 10 \%$ for all methods.

(A color version of this figure is available in the online journal.)

1. PSF type. For the best performing methods we find a trend that both ellipticity and size are estimated more accurately for the Airy PSF than for the Moffat PSF.

2. Addition of Kolmogorov power. For each set combination where both Moffat and Moffat-plus-Kolmogorov power are available (e.g., the 4 arm + masks) we find evidence for methods performing less well with the addition of Kolmogorov power (see also Figures 3-5). In Figure 6 we also show the impact of adding a Kolmogorov power spectrum to a set that uses an Airy PSF profile. We find that the addition of this random component degrades the residual ellipticity reconstruction by a factor of $\gtrsim 2-5$, but has less impact on size reconstruction, as expected, since the power is in ellipticity only. These results will necessarily depend on the amplitude of the assumed power spectrum, which will vary for each ground-based telescope, and knowledge/information about this is improving (e.g., Heymans et al. 2012). In addition, atmospheric turbulence also changes the PSF size, but we do not simulate this here. It is possible that, depending on the site and weather, the impact of turbulence may be weaker or stronger than that simulated for this study.

3. Masks. We show results for the mask variation in Figure 3. We find that for all methods the presence of diffraction spikes does not degrade the ability to measure the ellipticity of the PSF. For the Airy function the diffraction spikes act to increase the effective size of the PSF, which enables methods to measure the fractional error $\sigma\left(R^{2}\right) / R^{2}$ more accurately; but note that for a fixed $\sigma\left(R^{2}\right)$ a large size will decrease the fractional error by definition. For the Moffat PSF the diffraction spikes impact the size estimation significantly. We note, however, that this was a simple addition of a mask with no commensurate change in the variation of ellipticity or size across a field of view. Also the diffraction spikes contained low flux (only observable with the eye if one stacked all stars); higher signal-to-noise stars would change this. We leave an investigation of these effects to future work.

4. Number of stars. We find that all methods are only weakly dependent, or insensitive to the number of stars used to reconstruct the PSF in these simulations, except for those sets in which we include a Kolmogorov power spectrum where we find that a larger number of stars results in a better reconstruction for the best methods (see Figure 4). This indicates that PSFs with spatial power on smaller scales require more stars for a particular reconstruction accuracy than PSFs without power on small spatial scales.

5. Size of PSF. For the Airy profile we find that the larger the PSF the more accurately its size can be measured; for the Moffat we find a weak dependence with size. This is understandable because a larger PSF is better sampled and hence the size is easier to measure. This statement applies for PSFs with sizes 1.5 and 3 pixels in this study, but is certainly not true in all generality: once the sampling is better than critical, other factors (e.g., noise) take over (which our results also support). We also stress that an increase of the size of the PSF relative to the apparent size of galaxies will cause the galaxies to be less well-resolved, losing information and placing greater demands on shape measurement (Paulin-Henriksson et al. 2008). Also with the simulations presented, the impact of sampling on weak lensing shape measurement was not tested, only the performances of the PSF interpolation methods. We show results for the PSF size variation in Figure 5. When trading requirements of PSF model residuals against 
requirements for resolution (i.e., the absolute size of the ellipticity and PSF) such behavior should be noted.

6. Telescope parameters. We show results for the PSF size variation in Figure 7 . In varying the telescope parameters in the Jarvis et al. (2008) model we change the fiducial parameters respectively $\left(a_{0}=0.014, d_{0}=-0.006, c_{0}=\right.$ $-0.010)$ to $a_{0}=-0.011, d_{0}=0.009$, and $c_{0}=-0.011$, i.e., an opposite astigmatism, a positive de-focus, and a $10 \%$ increased coma. We find that methods in this experiment were not affected by the change in de-focus, but performed better with the change in these astigmatism and coma parameters.

We discuss each method individually in the Appendix.

\section{CONCLUSIONS}

This paper presents the first blind simulation challenge aimed at testing optical PSF reconstruction methods. Simulations were generated in which participants were presented with a spatially varying PSF, sparsely sampled by stars, and asked to reconstruct the PSF at non-star positions. The competition, the GREAT10 Star Challenge, attracted 30 submissions from 9 teams; several of these teams were from non-astronomy backgrounds. The simulation presented participants with 27,500 stars over 1300 images subdivided into 26 sets, where in each set a category change was made in the type or spatial variation of the PSF. The simulations were intentionally simplistic so as to present the problem in an approachable way; in particular, the spatial variation of the PSF and the form of the PSF use simple analytic functions. In addition, only spatial variation, not temporal variation, was tested; hence these results should not be used to make specific statements about any particular experiment but should provide a benchmark with which methods can be tested and improved. ${ }^{22}$

In this paper we analyze the submissions by testing how well each one can measure the ellipticity and size of the PSF. We quantify this as the inverse variance in the modeled PSF in each image for ellipticity and sized squared—-defined using weighted quadrupole moments. This study was motivated by a desire to find methods that will be of use for weak gravitational lensing, where the PSF must be reconstructed to high accuracy (PaulinHenriksson et al. 2008, 2009) at galaxy positions, but these results should also be of more general interest for any science case that analyzes galaxy images with optical data.

The submissions, and this paper, present a snapshot of any methods' ability to model the PSF. Due to the nature of the competitive blind submissions, post-challenge tuning of methods, which may yield significant improvements for any given method over the results presented here (see Gentile et al. 2013, for example), were not investigated. Each method submitted is summarized in the Appendix. We can, however, make some general statements about regimes in which methods tend to perform well or poorly when run in a blind way.

The functional form of the PSF was either a Moffat function or an Airy function, the spatial variation of the PSF was modeled using the analytic function given in Jarvis et al. (2008). In addition we optionally included diffraction spikes ( + or $*$ forms), changed the PSF size (from 3.0 pixels to 1.5 or 6.0 pixels), changed the number of stars (from 1000 to 500 or 2000), and added an atmospheric turbulence pattern in ellipticity (with a Kolmogorov power spectrum). To summarize the conclusions we find the following.

\footnotetext{
${ }^{22}$ Data is available for download here http://great.roe.ac.uk/data/solutions/.
}

1. The best methods can reconstruct the PSF with an accuracy of $\sigma(e) \approx 2.5 \times 10^{-4}$ and $\sigma\left(R^{2}\right) / R^{2} \approx 7.4 \times 10^{-4}$ over all sets.

2. Methods that performed poorly did so in part because the functional form of the PSF was not modeled correctly (in particular the Airy function).

3. Smaller PSFs were more difficult to model than larger PSFs for the Airy function, but we add a caution that this does not mean larger PSFs are better for weak lensing because information on a target object is lost; instead this means that well sampled PSFs are better for weak lensing.

4. Diffraction spikes caused the size of Moffat PSFs to be modeled less accurately, but Airy PSFs more accurately, due to the increase in the effective size.

5. The addition of atmospheric Kolmogorov power (equivalent to short exposure PSFs; see Heymans et al. 2012) made ellipticity and size reconstruction less accurate by a factor of $\gtrsim 2-5$ for all methods. We add the caveat that the temporal nature of varying PSFs was not investigated, therefore methods such as cross-correlation between sequential images, which could potentially improve modeling, were not investigated.

For subsequent blind PSF modeling challenges the realism of the temporal and wavelength dependent nature of PSF variation could be included, and the simulations could be tailored to specific experiments.

Modeling the PSF is of critical importance in efforts to understand the nature of dark energy and dark matter using weak gravitational lensing where any inaccuracy in the modeled PSF can cause biases, and increased errors of cosmological parameters of interest. To address this crucial open problem this initial presentation of a blind PSF reconstruction challenge will hopefully provide a benchmark upon which methods can continue to be refined and tested.

T.D.K. is supported by a Royal Society University Research Fellowship, and was supported by a Royal Astronomical Society 2010 Fellowship and the University of Edinburgh for some of this work. B.R. and C.H. acknowledge support from the European Research Council in the form of a Starting Grant with numbers 24067 (B.R.) and 240185 (C.H.). R.M. is supported by a Royal Society University Research Fellowship. D.G. was supported by SFB-Transregio 33 "The Dark Universe" by the Deutsche Forschungsgemeinschaft (DFG) and the DFG cluster of excellence "Origin and Structure of the Universe" and thanks Gary Bernstein and Stella Seitz for helpful discussions. M.Ge., G.C., and G.M. are supported by the Swiss National Science Foundation (SNSF). G.L. thanks Wei Cui for useful discussions. G.L. and B.X. were supported in part by the U.S. Department of Energy through Grant DE-FG02-91ER4068 and G.L. is also supported by the one-hundred talents program of the Chinese Academy of Sciences (CAS). M.K. thanks Liping Fu. This work was funded by a EU FP7 PASCAL 2 Challenge Grant. Workshops for the GREAT10 Challenge were funded by the eScience STFC Theme and by NASA JPL, and hosted at the eScience Institute Edinburgh and by IPAC Caltech, Pasadena. We thank Francesca Ziolkowska, Harry Teplitz, and Helene Seibly for local organization of the workshops. We thank the GREAT10 Advisory team, co-authors of the GREAT10 handbook (Kitching et al. 2012a), for discussions before and after the challenge.

Contributions: All authors contributed to the writing and analysis presented. T.D.K. was PI of GREAT10, defined and 
created the simulations, and lead the analysis. T.D.K., B.R., M.G., C.H., R.M. were active members of the GREAT10 team during 2010 December to 2011 September and after the challenge. B.R. created the FITS image simulation code. M.Ge., F.C., G.M., D.G., M.K., K.G., A.S., A.M., G.L., B.X. submitted entries to the GREAT10 Star Challenge. D.W. maintained the GREAT10 leaderboard and processed submissions with T.D.K. during the challenge.

\section{APPENDIX}

\section{DESCRIPTION OF METHODS}

Here we include a brief description and references for each of the methods submitted to the challenge.

Several methods use the name "Kriging," which is in fact the same method as Gaussian process regression, the method submitted for the methods MoffatGP; Kriging is a different term which has been in use in the geostatistics field but all are types of Gaussian processes.

\section{A.1.PSFEx (Gruen)}

PSFEx uses version 3.9.1 of the PSFEx software (Bertin 2011). The method models the PSF using a functional basis, the coefficients of which are allowed to vary with a polynomial dependence on the position in the field. Details of the configuration can be found in the PSFEx manual. ${ }^{23}$ For the GREAT10 submission, the functional basis is chosen to be a sub-pixel grid, from which PSF images on the input pixel scale are produced using Lanczos interpolation of order 4 . In order to improve configuration parameters, the $P$ metric is calculated on stars reserved from the fit. For this a Gaussian weight function with much smaller scale than in the final analysis ( 3 pixel FWHM) is used in order to suppress the noise in the images. The spatial variation was chosen to be of order 8 (4) on the sets with (without) atmospheric Kolmogorov power and the size of the sub-pixel grid to be $1 / 4.7$ of the PSF FWHM on all sets except the very undersampled sets 6,14 and 26, where a scale of 0.25 pixels is used instead. Note that these choices were made without knowledge of the true properties of the sets.

\section{A.2. $P C A+K r i g i n g ~(L i, X i n)$}

The basic idea of this method is to find the principal components of an ensemble of stars in an image. To find the correct principal components (PCs), stars first had to be centered: for this step a fast algorithm ( $\mathrm{Li}$ et al. 2012) was used to locate the centroid for each star and then an ordinary Kriging fitting algorithm was used to reproduce the star, whose center was exactly located at the center of the stamp grid. Each star was represented by 5 PCs, with five corresponding coefficients. According to the noise in the star stamp, an additional Gaussian noise component was included in each pixel and the corresponding coefficients were re-evaluated in 10 realizations; this helped us to estimate the uncertainties for each coefficient of PCs and each star. An ordinary Kriging fitting process was then used to predict the value of each coefficient at the asked positions and the new stars were composed of these 5 PCs with the predicted coefficients.

\section{A.3. Gaussianlets (Li, Xin)}

Gaussianlets is a simplified version of shapelets without any angular components, i.e., there are no shapelets with $m \neq 0$. The

\footnotetext{
23 http://www.astromatic.net/software/psfex
}

ellipticity of each star was calculated at the first step, then the size of each star $R_{i}$ was estimated quickly using the algorithm described in Li et al. (2012). One set of unique Gaussianlets with a maximum order $n_{\max }=4$ were created with $R=\left\langle R_{i}\right\rangle$. These Gaussianlets are circularly symmetric and were then reshaped into elliptical profiles according to the ellipticities that were measured in the first step to fit an individual star. The coefficients of Gaussianlets were calculated by minimizing a chi-square function. Finally each star was described by 7 parameters, $e_{1}$, $e_{2}$ and the five coefficients of gaussianlets. Ordinary Kriging interpolation was then used to predict these seven parameters at the asked positions. To reproduce the expected virtual star, the gaussianlets were reshaped according to $e_{1}$ and $e_{2}$ and were added up together according to their coefficients.

\section{A.4. B-Splines (Gentile, Courbin, Meylan)}

The B-Splines method, like the IDW, RBF, and Kriging schemes also described in this paper, uses the same underlying PSF estimation scheme that consists of the following stages. First, an elliptical Moffat profile is fitted to each star at a known position. Fitting is performed using a custom-developed minimizer based on an "adaptive cyclic coordinate descent algorithm." This minimizer is also used in the gfit galaxy shape measurement method described in Kitching et al. (2012a). Second, an analysis is performed of the spatial distribution of each Moffat parameter across the image. Third, a spatial interpolation scheme is adopted (here B-Splines) to predict the values of each Moffat parameter $p$ at asked positions. Finally, pixelized star images are reconstructed at asked positions based on the interpolated Moffat profile.

B-Splines perform a spatial interpolation of individual Moffat PSF parameters using the bivariate basis-spline algorithm described in Dierckx $(1980,1995)$ and implemented in the Python SciPy interpolate module. The main parameters affecting the interpolation are the degree of the spline, the number of knots, and a smoothing factor. A third-order spline was used but the algorithm was allowed to automatically optimize the number of knots and the smoothing factor.

A more thorough description of B-Splines, as well as the IDW, RBF, and Kriging interpolation methods can be found in Gentile et al. (2013).

\section{A.5. Inverse Distance Weighting (IDW) (Gentile, Courbin, Meylan)}

The IDW interpolation algorithm (Shepard 1968) is used to interpolate the Moffat parameters of the fitted PSF (see B-splines). Weights are allocated to the stars or parameters to interpolate. The closer the observations from a target location, the greater the weight ascribed to them. The estimated value of the parameter at the target point is a weighted sum of the values of all neighboring observations considered. The weighting power $\gamma$ determines how fast the weights tend to zero as distances increase. The Star Challenge results were obtained with $\gamma=2$ with a neighborhood size between 5 and 15 pixels depending on the density of stars.

\section{A.6. Radial Basis Function (RBF and RBF-thin) (Gentile, Courbin, Meylan)}

The RBF and RBF-thin methods make use of Radial Basis Functions to predict the values of the PSF parameters at non-star positions. As in B-splines the PSF is approximated by a Moffat profile. A Radial Basis Function (Buhmann 2003; 
Press et al. 2007), is a radially symmetric, real-valued function, whose value at a target location only depends on the distance to some other point. The prediction at a target location is based on the weighted sum of the RBFs evaluated in a neighborhood centered at that location.

The RBF and RBF-thin methods respectively use the linear and thin-plate functions. Their implementation is based on the interpolation function available in the Python SciPy interpolate module with a neighborhood size between 25 and 30 pixels. For the submission to the challenge, smoothing was disabled, i.e., exact interpolation was used where the PSF reconstructed at known positions should be exactly the input data.

\section{A.7. Kriging (Gentile, Courbin, Meylan)}

Ordinary Kriging (e.g., Waller \& Gotway 2004; Webster \& Oliver 2007) is used to interpolate PSF parameters (Moffat profiles as in B-splines) across the PSF field. For the Star Challenge, a unique implementation was created in Python for greater flexibility and control of the algorithm. In this version, no attempt was made to correct for any spatial anisotropy or drift found in the data.

The experimental variograms were fitted using the Levenberg-Marquardt (Levenberg 1980; Marquardt 1963) fitting function from the SciPy optimize module. The program dynamically selects the theoretical variogram models and parameters that produce the best fit. The area used for interpolation is a circular area with a radius between 700 and 1000 pixels from the center of the $4800 \times 4800$ PSF field. Lag distances were selected in the range 100 pixels $\leqslant h \leqslant 300$ pixels depending on the image and the PSF model parameter to estimate. The number of observations $N$ to include in the interpolation neighborhood was typically $5 \leqslant N \leqslant 20$ depending on the image star density. As a rule of thumb a tolerance was adopted for the distances $\Delta h \approx h / 2$ and angles $\Delta \theta=22.5$.

\section{A.8. IDWStk (Gentile, Courbin, Meylan)}

The IDWStk method experimented with an algorithm whereby the star postage stamp to reconstruct at asked position is estimated by stacking the pixels of nearby surrounding stamps located at known positions. Each pixel carries a weight that depends on its distance to the location where reconstruction has to take place. These weighting factors are calculated using IDW. For the Star Challenge, the number of surrounding nearby stars in the stacking was typically 10.

\section{A.9. MoffatGP (Georgatzis, Mariglis, Storkey)}

Predictions for the parameters of the Moffat model were made at the asked positions (with their corresponding offsets) and then the star images at test positions were reconstructed using the Moffat function (generating a $48 \times 48$ image for each star patch). This was done by finding five coefficients per star patch (position, ellipticity, and size) which were then used as training outputs for the regression method. Regression was performed using the Gaussian Process (GP) framework on an augmented input space. Along with the stars' center locations, for each star patch a distinct variable the offset of the star center from the bottom left corner of the center pixel was isolated, and provided as an additional input to the GP. The neural network covariance function Rasmussen \& Williams (2006) was chosen to encode correlations between data points. Predictions for the coefficients were made at the asked positions (with their corresponding offsets) and then the star images at test positions were reconstructed using the Moffat model (generating a $48 \times$ 48 image for each star patch). The method is described in more detail in Georgatzis (2011).

\section{REFERENCES}

Albrecht, A., Bernstein, G., Cahn, R., et al. 2006, arXiv:astro-ph/0609591

Bacon, D., Massey, R. J., Refregier, A. R., \& Ellis, R. S. 2003, MNRAS, 344, 673

Bartelmann, M., \& Schneider, P. 2001, PhR, 340, 291

Bergé, J., Price, S., Amara, A., \& Rhodes, J. 2012, MNRAS, 419, 2356

Bernstein, G., \& Jarvis, M. 2002, AJ, 123, 583

Bertin, E. 2011, adass, 442, 435

Bridle, S., Balan, S. T., Bethge, M., et al. 2010, MNRAS, 405, 2044

Buhmann, M. D. 2003, Radial Basis Functions: Theory and Implementations, Vol. 12 (Cambridge: Cambridge Univ. Press), 274

Chang, C., Kahn, S. M., Jernigan, J. G., et al. 2012, MNRAS, in press (arXiv:1206.1378)

Cropper, M., Hoekstra, H., Kitching, T., et al. 2012, MNRAS, submitted (arXiv:1210.7691)

Cypriano, E., Amara, A., Voigt, L., et al. 2010, MNRAS, 405, 494

Dierckx, P. 1980, An Algorithm for Surface Fitting with Spline Functions (Katholieke Univ. Leuven)

Dierckx, P. 1995, Curve and Surface Fitting with Splines, Monographs on Numerical Analysis (Oxford: Oxford Univ. Press)

Gentile, M., Courbin, F., \& Meylan, G. 2013, A\&A, 549, A1

Georgatzis, K. 2011, Master's thesis, Univ. Edinburgh

Heymans, C., Rowe, B., Hoekstra, H., et al. 2012, MNRAS, 421, 381

Heymans, C., Van Waerbeke, L., Bacon, D., et al. 2006, MNRAS, 368, 1323

Hoekstra, H. 2004, MNRAS, 347, 1337

Hoekstra, H., \& Jain, B. 2008, ARNPS, 58, 99

Hoekstra, H., Yee, H., \& Gladders, M. 2004, ApJ, 606, 67

Hu, W. 1999, ApJ, 522, L21

Jain, B., Jarvis, M., \& Bernstein, G. 2006, JCAP, 0602, 001

Jarvis, M., \& Jain, B. 2005, ApJ, submitted (astro-ph/0412234)

Jarvis, M., Schechter, P., \& Jain, B. 2008, arXiv:0810.0027

Kitching, T., Balan, S., Bernstein, G., et al. 2012a, AoAS, 5, 2231

Kitching, T. D., Balan, S. T., Bridle, S., et al. 2012b, MNRAS, 423, 3236

Kitching, T. D., Rhodes, J., Heymans, C., et al. 2012c, NewAR, submitted (arXiv:1204.4096)

Kuijken, K. 2006, arXiv:astro-ph/0610606

Levenberg, K. 1944, QApMa, 2, 164

Li, G., Xin, B., \& Cui, W. 2012, arXiv:1203.0571

Marquardt, D. W. 1963, J. Soc. Ind. Appl. Math., 11, 431

Massey, R., Heymans, C., Bergé, J., et al. 2007, MNRAS, 376, 13

Massey, R., Hoekstra, H., Kitching, T., et al. 2013, MNRAS, 429, 661

Massey, R., Kitching, T., \& Richard, J. 2010, RPPh, 73, 086901

Moffat, A. F. J. 1969, A\&A, 3, 455

Papageorgiou, A. M. 2011, Master's thesis, Univ. Edinburgh

Paulin-Henriksson, S., Amara, A., Voigt, L., Réfrégier, A., \& Bridle, S. 2008, A\&A, 484, 67

Paulin-Henriksson, S., Réfrégier, A., \& Amara, A. 2009, A\&A, 500, 647

Plazas, A. A., \& Bernstein, G. M. 2012, PASP, in press (arXiv:1204.1346)

Press, W. H., Teukolsky, S. A., Vetterling, W. T., \& Flannery, B. P. 2007, Numerical Recipes: The Art of Scientific Computing (3rd ed.; Cambridge: Cambridge Univ. Press)

Rasmussen, C. E., \& Williams, C. K. I. 2006, Gaussian Processes for Machine Learning. Adaptive Computation and Machine Learning (Cambridge, MA: MIT Press)

Réfrégier, A. 2003, ARA\&A, 41, 645

Rhodes, J., Massey, R. J., Albert, J., et al. 2007, ApJS, 172, 203

Rowe, B. 2010, MNRAS, 404, 350

Schrabback, T., Hartlap, J., Joachimi, B., et al. 2010, A\&A, 516, 63

Shepard, D. 1968, in ACM 68: Proc. 1968 23rd ACM national conference, ed. R. B. Blue, Sr. \& A. M. Rosenberg (New York: ACM), 517

van Waerbeke, L., Mellier, Y., \& Hoekstra, H. 2005, A\&A, 429, 75

Voigt, L., Bridle, S., Amara, A., et al. 2012, MNRAS, 421, 1385

Waller, L., \& Gotway, C. 2004, Applied Spatial Statistics for Public Health Data, Wiley Series in Probability and Statistics (New York: Wiley)

Webster, R., \& Oliver, M. 2007, Geostatistics for Environmental Scientists, Statistics in Practice (New York: Wiley)

Weinberg, D. H., Mortonson, M. J., Eisenstein, D. J., et al. 2012, arXiv:1201.2434

Wells, D. C., Greisen, E. W., \& Harten, R. H. 1981, A\&AS, 44, 363 